J. Lake Sci.(湖泊科学) , 2008, 20(1): 13-20

http://www.jlakes.org. E-mail: jlakes@niglas.ac.cn

(C)2008 by Journal of Lake Sciences

\title{
稳定同位素技术应用于水域食物网的方法学研究进展"
}

\author{
曾庆飞 ${ }^{1,2}$, 孔繁翔 ${ }^{1}$, 张恩楼 ${ }^{1}$, 钱善勤 ${ }^{1,2}$ \\ (1: 中国科学院南京地理与湖泊研究所湖泊与环境国家重点实验室，南京 210008) \\ (2: 中国科学院研究生院, 北京 100049)
}

摘 要: 稳定同位素技术是揭示食物网中物质的循环路径和探究消费者、生产者间的营养关系的重要方法. 但是, 不同的样品 处理方法可能引起同位素比值的变动, 使得食物网模型的建立和各研究结果间的相互比较存在一定的困难. 样品的预处理主 要分为四个环节, 即保存、分离、酸化和干燥. 在水域生态系统中, 水体中的颗粒/溶解性有机物质、细菌、浮游动物、藻类、 水生植物、鱼类及底栖动物等采集后, 通过分离纯化, 得到目的样品, $-20^{\circ} \mathrm{C}$ 冷冻保存. 对于含有无机碳酸盐的生物样品, 例如 沉积物、含有钙质结构的无脊椎生物、部分浮游生物等需要酸化处理以排除无机碳对 $\delta^{13} \mathrm{C}$ 测定的影响, $1 \mathrm{~mol} / \mathrm{L} \mathrm{HCl}$ 滴加可以 达到很好的去除效果. 对于生物样品 $\delta^{15} \mathrm{~N}$ 的测定则不需要酸化处理. 最后, 样品经冷冻干燥或 $40-70^{\circ} \mathrm{C}$ 低温烘干以备稳定同 位素的测定. 另外, 用福尔马林、乙醇等保存在博物馆里的样品, 对于长时间尺度上食物网的重建具有重要的生态学意义. 本 文系统总结了不同研究中样品处理过程的差异, 分析其干扰机制, 为进一步的方法学研究奠定了基础.

关键词: 稳定同位素; 水域食物网; 方法学

\section{Assessment of sample processing methods for stable isotope analysis of aquatic food webs}

\author{
ZENG Qingfei $^{1,2}$, KONG Fanxiang $^{1}$, ZHANG Enlou ${ }^{1}$ \& QIAN Shanqin ${ }^{1,2}$ \\ (1: State Key Laboratory of Lake Science and Environment, Nanjing Institute of Geography and Limnology, Chinese Academy of \\ Sciences, Nanjing 210008, P.R China) \\ (2: Graduate School of Chinese Academy of Sciences, Beijing 100049, P.R.China)
}

Abstract: Stable isotope analysis is commonly used in studying flows of mass and energy through food webs and trophic relationships in aquatic ecosystems. However, different sample processing methods can influence the measurement of these stable isotope rates, which may result in errors in the resulting food web models and the comparing results between different studies. In particular, errors may result from four different sources, that is, preservation, separation, acidification and dehydration. The collectted particulate/dissolved organic matter, bacteria, zooplankton, algae, hydrophyte, fish and zoobenthos were rinsed with de-ionized water to clean off epibionts, and then stored at $-20^{\circ} \mathrm{C}$. Acidification by adding $1 \mathrm{~mol} / \mathrm{L} \mathrm{HCl}$ drop-by-drop was needed for carbon isotope analysis in samples of sediment organic matter, invertebrates with calcareous structures, and plankton. For nitrogen analysis, acidification should be avoided. Finally, dehydration was required by the analytical methods used in the determination of isotopic abundance. Both freeze-drying and drying at $40-70^{\circ} \mathrm{C}$ were acceptable. In addition, materials preserved with formalin and ethanol stocks was suitable for current ecological applications of isotopic analysis and open up the possibility to reconstruct food webs and biogeochemical changes at scales of tens or hundreds of years. In this review we summarize different sample processing prior to the analytical determination of stable isotope ratios and the influence mechanism of some processing methods, which are fundamental for further methodology research.

Keywords: Stable isotope; aquatic food webs; methodology

近年来, 稳定同位素技术广泛地应用于水域食物网中, 用于揭示有机物质的循环路径和探究消费者

* 国家重点基础研究发展计划(973)项目(2002CB412305)、国家自然科学基金项目(40471045)和中国科学院百人计划联合资助. 2007-04-29 收稿; 2007-08-19 收修改稿. 曾庆飞, 女, 1979 年生, 博士研究生; E-mail: zq-fly@sohu.com. 
之间的营养关系 ${ }^{[1-3]}$. 利用生物天然碳氮稳定同位素可以有效地揭示其有机物来源、消费者的食物组成以 及各生物在食物网中所处的营养级. 氮同位素在不同营养级有大约 3.4\% 的富集度, 通常用来指示生物所 处的营养级; 消费者的碳同位素和其食源基本相似，平均富集在 $0.1 \% 0-0.4 \%$ 之间，是食物来源良好的示 踪指标 ${ }^{[4]}$. 但是, 在同位素分析前, 有关样品的前处理方法在各研究中却存在很大的差异, 直接影响了结 果的准确性和客观性. 这使得稳定同位素比值在各研究间的相互比较存在很大的困难，也不便于联合各 研究中不同生物的同位素比值去构建完整的食物网模型 ${ }^{[5]}$.

通常, 差异来源主要有以下四个环节, 即样品的保存、分离、酸化和干燥. 在样品同位素测定前, 特别 是野外采集的样品, 需要采取一定保护措施诸如 $-20^{\circ} \mathrm{C}$ 冷冻、直接干燥、盐浸、福尔马林或乙醇浸泡等以防 止有机物质变质. 同时, 对于食物网中较小生物要进行必要的分离与纯化. 某些生物样品含有颗粒性无机 碳, 干扰有机体碳同位素的测定, 需要采取必要的酸化措施以除去碳酸盐的影响. 最后, 干燥样品以达到测 定的要求. Bosley 和 Wainright ${ }^{[6]}$ 比较了经福尔马林、福尔马林/乙醇和饱和氯化录保存几个月的样品, 发现 它们引起了 $\delta^{15} \mathrm{~N}$ 的显著增加和 $\delta^{13} \mathrm{C}$ 的降低, 最后指出冷冻是保存样品的最好方法. Kaehler 和 Pakhomov ${ }^{[7]}$ 也发现冷冻和低温烘干没有引起同位素比值的显著变化. 而 Lorrain 等 ${ }^{[8]}$ 的研究表明, 冷冻引起了 $\delta^{15} \mathrm{~N}$ 的不 确定性波动, 所以他认为直接干燥是保存样品的最好方法. 同时他发现稀酸浸泡不能有效去除有机体内的 碳酸盐, 浓酸熏蒸才是最行之有效的方法. Jacob 等[9]则认为稀酸滴加就可以达到去除碳酸盐干扰的目的.

可见, 不同的研究得到的结论也不尽相同. 至今, 对于同位素测定的前处理方法还没有一个统一的 标准. 而国内在稳定同位素技术在食物网应用方面还处在起步阶段，系统性的方法学探讨还鲜有报道 ${ }^{[10]}$. 因此, 本文通过系统比较不同预处理方法所引起的碳氮稳定同位素比值的差异, 分析其干扰机制, 为不 同样品的处理方法提供参考依据，以期能为标准的预处理方法的建立做出贡献，从而推动稳定同位素技 术在水域生态系统中的应用.

\section{1 样品保存}

\section{1 生物样品}

妥善的保存方法是保证样品同位素比值精度的一个首要条件. 目前, $-20^{\circ} \mathrm{C}$ 冷冻是最理想的样品存諚， 方法 ${ }^{[5-6,11]}$. 但是野外操作很难达到这一要求. 因保存在博物馆和地壳中的生物标本对于长时间尺度上的 食物网的重建及生物地球化学过程的研究具有重要的参考价值 ${ }^{[5,12]}$, 所以探讨不同保存方法对稳定同位 素比值的影响意义重大.

生物样品通常的保存方法是浸泡在 $10 \%$ 福尔马林 ${ }^{[5-6]}$, 或者是 $75 \%$ 的乙醇 ${ }^{[5]}$, 更为标准的方法是 $10 \%$ 福尔马林浸泡 2 周, 随后转移到 $70 \%-90 \%$ 的乙醇中 ${ }^{[6,13]}$. 相比于冷冻保存的样品, 福尔马林和乙醇引起了 氮稳定同位素的显著增加，变化幅度在 $0.5 \% 0-1.4 \%$ 之间 ${ }^{[6]}$. 而Sarakinos 等 ${ }^{[5]}$ 研究表明防腐剂对 $\delta^{15} \mathrm{~N}$ 的影响 很小. 由于两种保存剂均不含 $\mathrm{N}$ 元素, 可能的原因就是保存过程中组织水解, 物质溶出造成的. 同时, 他 还发现福尔马林保存对碳同位素比值的影响较为显著, 较冷冻样品平均贫化 $1.65 \%$, 而乙醇固定的影响 程度较小 $(-0.02 \% 0 \pm 0.24 \%$ ). 这主要是由于二者对样品的影响机制不同而引起的. 首先, 样品在保存过程 中物质的选择性流失. 乙醇水解脂类, 而福尔马林水解蛋白质 ${ }^{[6]}$. 脂类的碳同位素较蛋白质更为贫化 ${ }^{[14]}$, 所 以用福尔马林保存的样品由于蛋白质水解, 脂类相对聚集导致了整体碳同位素比值较低. 另外，福尔马 林和乙醇都是含有碳元素的化合物, 本身同位素比值的差异可能会引起浸泡其中的样品的差异 ${ }^{[5]}$. 所以 同位素测定前, 样品充分润洗后, 建议在去离子水中浸泡 $48 \mathrm{~h}^{[13]}$. Vander Zanden等 ${ }^{[12]}$ 统计了防腐剂对 18 种 鱼类和无脊椎动物同位素比值的影响, 发现福尔马林保存引起了 ${ }^{13} \mathrm{C}$ 的降低, 校正系数为 $-1.28 \% \pm 0.22 \%$; 而对 $\delta^{15} \mathrm{~N}$ 的影响波动较大, 其中鱼类为 $0.53 \% 0 \pm 0.15 \%$, 无脊椎动物为 $-0.18 \% 0 \pm 0.29 \%$. 可见, 虽然防腐保 存引起了同位素比值的变化, 但相对于生物转化的同位素分馏还较小, 通过系数矫正, 重建食物网结构, 以揭示长时间尺度上食物网结构的历史变化 ${ }^{[5,11-13]}$.

保存剂的保存时间长短对同位素比值也有影响. 保存6周对氮同位素影响不大, 再长, 变化的可能性 增大 ${ }^{[13]}$. Mullin等 ${ }^{[15]}$ 发现保存在福尔马林中的浮游动物两年内氮同位素差异不大. 样品保存还可以采用

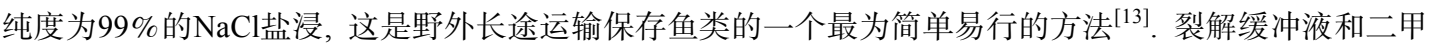


基亚砜等用于基因分析的保护液会影响碳氮稳定同位素, 所以不建议使用 ${ }^{[16]}$.

如果条件允许, Lorrain等 ${ }^{[8]}$ 和Rau等 ${ }^{[17]}$ 认为直接干燥是进行样品长期保存的首选方法. 因为他的研究 表明, 将 $\mathrm{GF} / \mathrm{F}$ 滤膜收集的 $<200 \mu \mathrm{m}$ 颗粒性有机物 $(\mathrm{POM})$ 在 $-20^{\circ} \mathrm{C}$ 保存后相对于直接干燥的会引起氮同位素 比值的显著变化和标准偏差的增加. 但是, Bosley ${ }^{[6]}$ 研究了褐虾肉直接新鲜低温干燥, $-80^{\circ} \mathrm{C}$ 冷冻保存几天 后再低温干燥 $\left(55^{\circ} \mathrm{C}\right)$ 和冷冻干燥三种方法对稳定同位素比值的影响, 发现均没有引起 $\delta^{13} \mathrm{C}$ 和 $\delta^{15} \mathrm{~N}$ 的显著 差异 $\left(\delta^{13} \mathrm{C}<0.3 \% ; \delta^{15} \mathrm{~N}<0.2 \%\right)$.

\section{2 无机样品}

水体中溶解态无机碳(DIC)是浮游植物光合作用的主要碳源. 目前, 国际间对于海水中 DIC 同位素的 测定, 缺乏一个可供共同比较的标准样品, 故各实验室之间的测定结果, 是否能直接加以比较, 备受质 疑. DIC 测定所需的水样以加人饱和氯化永溶液的 $350 \mathrm{ml}$ 透明的 BOD 瓶密封 ${ }^{[18]}, 4-7^{\circ} \mathrm{C}$ 冷藏保存. Cole ${ }^{[19]}$, Pace ${ }^{[20]}$, Kritzberg ${ }^{[1]}$ 等则是将用 $\mathrm{H}_{2} \mathrm{SO}_{4}$ 酸化到 $\mathrm{pH}=2$ 的水样用 $10 \mathrm{ml}$ 的 $\mathrm{N}_{2}$ 密封在 $100 \mathrm{ml}$ 的血清瓶中, 用于 同位素的测定. 关于样品储存时间方面, Atekwana ${ }^{[21]}$ 认为只要不超过 $43 \mathrm{~d}, \mathrm{DI}^{13} \mathrm{C}$ 比值变化不大. 林信吉 ${ }^{[18]}$ 的研究表明 $250 \mathrm{ml}$ 水样中 $0-2 \mathrm{ml}$ 饱和氯化录溶液添加量及一星期之三个月的储存时间, 对水样中 $\delta^{13} \mathrm{C}_{\mathrm{DIC}}$ 的 分析结果并无显著差异.

\section{2 分离}

\section{1 颗粒有机物(POM)和溶解性有机物(DOM)}

颗粒有机质和溶解性有机质是水体中两种重要的有机物质存在形式. 溶解性有机质主要来自浮游植 物光合作用的释放, 各类动物的排泄和分泌, 死亡生物残骸及粪便颗粒的分解, 动物摄食的释放等 ${ }^{[22]}$. 它是细菌二次生产进人微食物网的主要物质来源. 根据研究目的可以用不同孔径的滤膜将它们分开. 用 于同位素分析通常选择 Whatman $\mathrm{GF} / \mathrm{C}(1.2 \mu \mathrm{m})^{[23]}$ 和 $\mathrm{GF} / \mathrm{F}(0.7 \mu \mathrm{m})^{[24]}$ 玻璃纤维滤膜. 这样, 细菌、病毒和较 小的胶体颗粒就包含在溶解性有机物中. 理想情况, DOM样品应该是经孔径 $<0.2 \mu \mathrm{m}$ 的滤膜以去除滤液中 细菌的干扰 ${ }^{[25]}$. 由于浮游澡类的分解，在过滤过程中不可避免会在溶液中增加额外的 $\mathrm{DOM}^{[23]}$. 过滤应该 在低压 ${ }^{[26]}$ 或者是自然重力过滤 ${ }^{[27]}$. 过滤后的水样采用冷冻干燥或真空离心的方法浓缩成固体残渣 ${ }^{[24]}$. 在 设备条件无法满足的情况下, 相当的研究则采用 $40-70^{\circ} \mathrm{C}$ 低温烘干得到残渣用于同位素分析 ${ }^{[1,19-20]}$. 这种 方法所需的水样不多, 1-2L 即可达到同位素测定的要求. 对于盐分较高的海水通常采用切向旋转超滤系 统, 将至少 $20 \mathrm{~L}$ 水样浓缩为 $1 \mathrm{~L}$, 再 $30^{\circ} \mathrm{C}$ 低温蒸发至 $50 \mathrm{ml}$, 随后冷冻干燥 ${ }^{[29]}$.

POM同位素测量对滤膜的要求比较高, 必须是能耐高温的无机材料, 通常采用经 $450-525^{\circ} \mathrm{C}$ 灼烧 $4 \mathrm{~h}$ 的 GF/F玻璃纤维滤膜. 水样预先应采用一定孔径的滤网预滤以除去大型浮游动物和藻类 ${ }^{[1]}$. 也有研究选 择切向流旋转超滤系统收集 POM, 采用 $0.1 \mu \mathrm{m}$ 的聚砜中空纤维过滤器, 虽然认为这种方法可能有很大的 吸附损失 $(60 \%-70 \%)^{[30]}$.

\section{2 细菌}

细菌不仅是传统食物网中的分解者, 还是微食物网中的生产者, 对水域生态系统中的物质循环和能量 转化起重要的作用 ${ }^{[31]}$. 细菌稳定同位素测定的方法有: 细菌全细胞分析 ${ }^{[1,32]}$, 测定细菌呼吸的二氧化碳 ${ }^{[33]}$ 及提取细菌标志物如核酸 ${ }^{[29,34]}$, 肽聚糖细胞壁的氨基酸 ${ }^{[35]}$ 或脂肪酸中的极性脂 ${ }^{[36]}$ 等, 但这些方法各有利弊.

细菌标志物的提取避免了培养条件人为因素的干扰, 但同时也提取到了不想要的东西, 回收率低和 工作量大等缺点 ${ }^{[34]}$. 而且, 细菌标识物和整个细菌细胞碳同位素还是存在差异 ${ }^{[35]}$. 细菌全细胞稳定同位 素的测定是通过原位水样培养得到的. 利用细菌的繁殖速率快, $20-30^{\circ} \mathrm{C}$ 培养 $24-48 \mathrm{~h}$ 就可达到对数生长 期, 其生物量增殖 3-4 倍这一特性, Coffin 把细菌培养在不同碳同位素信号的有机培养基中, 发现其稳定 碳同位素比值同培养基质相似, 变化幅度在 $\pm 2 \%{ }^{[32]}$. 可见, 细菌原位水样培养的方法是可行的. Kritzberg 等 ${ }^{[1}$ 将该方法做了改进, 把细菌培养在原位采样点的透析袋中以保证细菌生长对藻类来源的 DOC 的依赖. 结果表明, 透析培养和玻璃容器培养二者差异不大, 细菌的碳同位素信号没有显著差异.

\section{3 浮游动物}

浮游动物是鱼类的重要饵料, 在食物网中起到承上启下的作用, 浮游动物的种群动态变化和生产力 
的高低, 对于水域生态系统结构功能以及生源要素循环都有着十分重要的影响 ${ }^{[37]}$. 根据其个体的大小, 通常分为微型浮游动物 $(20-200 \mu \mathrm{m})$, 中型浮游动物 $(200-2000 \mu \mathrm{m})$ 等. 对浮游动物的采集, 许多研究者采 用的浮游生物网孔径 $\geqslant 100 \mu \mathrm{m}^{[38]}$. 但如果网径 $>150 \mu \mathrm{m}$, 会导致桡足类无节幼体、原生动物、轮虫、软体 动物和多毛类环节动物的浮游幼虫等多种小型生物逃逸 ${ }^{[37]}$. 所以, 对于淡水生态系统建议采用标准的 25 号浮游生物网 $(64 \mu \mathrm{m})^{[39]}$, 而海洋浮游动物的采集网径通常较大. 采集到的浮游动物可以在预先经 Whatman GF/F过滤的原水中暂养以排空内容物, 但由于种种原因, 大部分的样品要在捕捞后 $5 \mathrm{~h}$ 内分拣和 冷冻保存 ${ }^{[40]}$.

\section{4 鱼类及底栖动物}

鱼类等大型浮游生物稳定同位素的测定较为简单, 去鳞片并在背部切去一小块肌肉, 干燥后即可 ${ }^{[28,41]}$. 对于带壳生物应根据研究目的, 如果是以带壳生物作为食物时, 在测量其同位素比值时应去掉外壳, 因 外壳较难被捕食者吸收; 当作为捕食者时应保留, 因外壳同位素同样来自食物 ${ }^{[42]}$. 脂类的聚集会引起组 织样品碳同位素的贫化，所以鱼类、软体动物等在进行同位素测定前建议进行去脂处理 ${ }^{[40]}$. 但是，是否去 脂和去脂处理的标准方法至今还没有统一的定论. Schlechtriem等 ${ }^{[43]}$ 研究了不同去脂方法, 如 $2: 1$ 氯仿: 甲 醇 $(\mathrm{V} / \mathrm{V})$, 甲醇, 汽油一乙醚等对鱼类及其食物的影响, 发现各处理方法没有引起 $\delta^{13} \mathrm{C}$ 显著差异. Murry等 ${ }^{[44]}$ 指出普通的去脂过程不仅提取了脂类，还水解了其它含氮化合物从而影响了 $\delta^{15} \mathrm{~N}$ 的精度，去脂处理造 成了组织样品碳氮稳定同位素比值的提高.

\section{5 藻类、水生植物}

藻类和高等的维管束植物是水域食物网主要的初级生产者, 也是浮游动物和草食性鱼类的重要食源. 水生植物的采集比较简单, 分析前刷去附着在上面的藻类和寄生虫, 清洗干净即可 ${ }^{[28]}$. 浓缩足量、较纯的 藻类进行稳定同位素分析, 通常的方法有光学显微镜人工挑选, 但这主要针对丝状藻和群聚藻, 特别是 在水华爆发(蓝藻、硅藻、褐藻等)出现优势藻种的时候较易采集 ${ }^{[39]}$. 底栖藻类可以通过光诱的方法使其 迁徙到水体中 ${ }^{[45]}$. 当水体中藻类含量较低时, 采用流式细胞仪分选浓缩 ${ }^{[46]}$, 或利用细胞色素标识物 Chl.a 进行同位素分析 ${ }^{[47]}$ 等，但所需的仪器昂贵，成本高. 因此，目前在进行消费者食物比例分析时，相当的研 究采用不同粒径的颗粒性有机物作为替代粗略估计消费者的食性组成 ${ }^{[2,24,48]}$. 这在很大程度上掩盖了摄 食者的选择性吸收, 因为颗粒性有机物是一个包含活性藻类、浮游生物残体和陆源碎屑的复杂混合物.

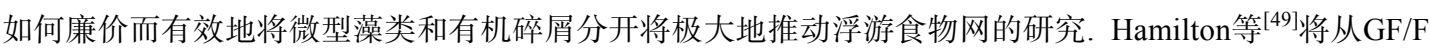
滤膜上收集到的颗粒有机物悬浮在密度为 $1.27 \mathrm{~g} / \mathrm{cm}^{3}$ 硅胶胶体中, 1000 转 $/ \mathrm{min}$ 离心 $10 \mathrm{~min}$, 溶液出现了分层 现象, 上层较轻的溶液主要为浮游藻类, 随后经 C: Chl. 和 C:N两个指标验证, 其分离效率达到 $75 \%$ 左右.

\section{3 酸化}

在分析沉积物和某些含有无机碳酸盐成分的生物机体时(甲壳类、软体动物等), 通常采用 $0.1-1 \mathrm{~mol} / \mathrm{L}$ 稀盐酸浸泡或浓盐酸 $(12 \mathrm{~mol} / \mathrm{L})$ 酸雾熏蒸的方法以除去其对碳稳定同位素比值的干扰. 有研究表明, 即使 很小的无机碳含量(小于有机质总碳的 $5 \%$ ), 就有可能引起 $\delta^{13} \mathrm{C}$ 比值 $1 \%$ 的增加 ${ }^{[17]}$. 但是, 在是否酸化问题 上还存在很大分歧.

Bosley 和Wainright ${ }^{[6]}$ 用 $1 \mathrm{~mol} / \mathrm{L} \mathrm{HCl}$ 溶液对碳酸钙含量较高的片刺褐虾和无机碳相当较少的幼比目鱼 进行酸化处理, 同未酸化的样品相比, 酸化没有引起 $\delta^{13} \mathrm{C}$ 的显著变化. 这同 Bunn 等 ${ }^{[42]}$ 的研究结果部分吻 合, 即用 $0.1 \mathrm{~mol} / \mathrm{L} \mathrm{HCl}$ 没有引起对虾和海草 $\delta^{13} \mathrm{C}$ 的显著变化, 但是氮同位素比值却随着盐酸浓度、酸化后 是否用去离子水润洗而发生显著的变化. Carabel等 ${ }^{[28]}$ 对食物网 13 种生物进行了细致的比较实验, 结果表 明酸化会降低沉积物(SOM), 颗粒有机质, 浮游生物和无脊椎动物的碳同位素比值, 而对氮同位素的影 响规律不一致. Lorrain等 ${ }^{[17]}$ 比较了盐酸浸泡和熏蒸两种方法的去碳酸盐效果, 发现用 $12 \mathrm{~mol} / \mathrm{L}$ 浓盐酸连续 熏蒸干燥的 GF/F滤膜 $(<200 \mu \mathrm{m} \mathrm{POM}) 4 \mathrm{~h}$, 无机碳去除效果明显，而且没有引起 $\delta^{15} \mathrm{~N}$ 的显著变化.

总体来说，相对于未酸化的样品，酸化处理引起了碳氮稳定同位素比值的降低(表1). 其中，对氮同 位素比值的影响可能是由于组织内不同的含氮化合物如核酸、氨基酸等在酸化过程中的溶出速率和溶出 量不同造成的, 所以建议氮同位素测定时不宜酸化处理 ${ }^{[28,50]} .10 \%$ 的 $\mathrm{HCl}$ 酸洗就有可能引起软组织 $\delta^{15} \mathrm{~N}$ 平 
均约 $3 \% 0$ 的降低 ${ }^{[51]}$. 其次, 在去酸清洗过程中可能造成部分有机碳的损失, 从而引起碳同位素比值的降低, 所以建议样品酸化后不进行去离子水清洗 ${ }^{[9,28]}$.

表 1 盐酸 $(\mathrm{HCl})$ 酸化对食物网生物稳定同位素比值 $\left(\delta^{13} \mathrm{C}\right.$ 和 $\left.\delta^{15} \mathrm{~N}\right)$ 的影响

Tab. 1 The effects of acidification on aquatic food web components stable isotope $\left(\delta^{13} \mathrm{C}\right.$ and $\left.\delta^{15} \mathrm{~N}\right)$ signatures

\begin{tabular}{|c|c|c|c|c|c|c|c|}
\hline \multirow{2}{*}{ 样品 } & \multicolumn{2}{|c|}{$0.1 \mathrm{~mol} / \mathrm{L} \mathrm{HCl}$} & \multicolumn{2}{|c|}{$1 \mathrm{~mol} / \mathrm{L} \mathrm{HCl}$} & \multicolumn{2}{|c|}{$12 \mathrm{~mol} / \mathrm{L} \mathrm{HCl}$ 熏蒸 } & \multirow{2}{*}{ 引用文献 } \\
\hline & $\delta^{13} \mathrm{C}$ & $\delta^{15} \mathrm{~N}$ & $\delta^{13} \mathrm{C}$ & $\delta^{15} \mathrm{~N}$ & $\delta^{13} \mathrm{C}$ & $\delta^{15} \mathrm{~N}$ & \\
\hline \multicolumn{8}{|l|}{ 碳含量较少 } \\
\hline 比目鱼 (P.americanus) & & & ns & $\mathrm{ns}$ & ns & $\mathrm{ns}$ & Bosley \& Wainright ${ }^{[6]}$ \\
\hline POM 膜 ${ }^{a}$ & -0.8 & 0.1 & & & -1.1 & -0.2 & Lorrain et al ${ }^{[8]}$ \\
\hline POM 膜 ${ }^{b}$ & -0.7 & 0.4 & & & -1.0 & -0.4 & Lorrain et $\mathrm{al}^{[8]}$ \\
\hline 无脊椎动物和鱼类 & & & -0.117 & -0.320 & & & Jacob et $\mathrm{al}^{[9]}$ \\
\hline 海菖蒲 (E. acoroides) & 0 & -1.8 & & & & & Bunn et $\mathrm{al}^{42]}$ \\
\hline 极北海带 (L. hyperborean) & & & -0.23 & -0.12 & & & Carabel et al ${ }^{[28]}$ \\
\hline 大眼鳕鱼 (Trisopterus luscus) & & & -0.02 & -0.28 & & & Carabel et al ${ }^{[28]}$ \\
\hline 章鱼 (Octopus vulgaris) & & & 0.06 & 0.58 & & & Carabel et al ${ }^{[28]}$ \\
\hline 欧洲螃蟹肌肉 (Polybius henslowii) & & & -0.74 & -0.02 & & & Carabel et al ${ }^{[28]}$ \\
\hline 均值 & -0.75 & -0.43 & -0.21 & -0.03 & -1.05 & -0.3 & \\
\hline 均方差 & 0.07 & 1.19 & 0.32 & 0.36 & 0.07 & 1.14 & \\
\hline \multicolumn{8}{|l|}{ 碳含量较多 } \\
\hline 片刺褐虾 (C.septemspinosa) & & & ns & ns & $\mathrm{ns}$ & $\mathrm{ns}$ & Bosley \& Wainright ${ }^{[6]}$ \\
\hline 对虾 (Metapenaeus spp.) & -0.3 & 2.9 & & & & & Bunn et al ${ }^{[42]}$ \\
\hline SOM & & & -3.99 & -0.32 & & & Carabel et al ${ }^{[28]}$ \\
\hline SOM 再悬浮处理 ${ }^{\mathrm{c}}$ & & & -0.05 & 0.79 & & & Carabel et al ${ }^{[28]}$ \\
\hline SOM 再悬浮处理 ${ }^{a}$ & & & -1.32 & -0.66 & & & Carabel et al ${ }^{[28]}$ \\
\hline SPOM 膜 & & & -3.67 & -0.73 & & & Carabel et al ${ }^{[28]}$ \\
\hline 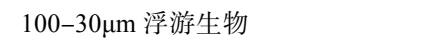 & & & -0.71 & 0.02 & & & Carabel et al ${ }^{[28]}$ \\
\hline $300-500 \mu \mathrm{m}$ 浮游生物 ${ }^{\mathrm{c}}$ & & & -0.27 & 0.26 & & & Carabel et al ${ }^{[28]}$ \\
\hline $300-500 \mu \mathrm{m}$ 浮游生物 ${ }^{\mathrm{a}}$ & & & 0.29 & -0.43 & & & Carabel et al ${ }^{[28]}$ \\
\hline 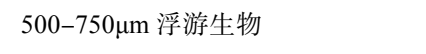 & & & -0.31 & 0.98 & & & Carabel et al ${ }^{[28]}$ \\
\hline 欧洲螃蟹整体 (Polybius henslowii) & & & -2.06 & -1.06 & & & Carabel et al ${ }^{[28]}$ \\
\hline 普白磨面螃蟹 (Necora puber) & & & -0.27 & 0.24 & & & Carabel et al ${ }^{[28]}$ \\
\hline 均值 & -0.3 & 2.9 & -1.17 & -0.05 & & & \\
\hline 均方差 & & & 1.46 & 0.64 & & & \\
\hline 总平均 & -0.6 & 0.4 & -0.87 & -0.05 & -1.05 & -0.3 & \\
\hline 总均方差 & 0.26 & 1.93 & 1.29 & 0.56 & 0.07 & 1.14 & \\
\hline
\end{tabular}

$\mathrm{a}: 60^{\circ} \mathrm{C}$ 低温干燥后酸化; b: $20^{\circ} \mathrm{C}$ 冷冻后酸化; c: 冷冻干燥后酸化; ns 代表差异不显著.

\section{4 干燥}

脱水是样品进行同位素分析的必要过程. 通常的干燥方法有真空干燥、冷冻干燥和 $40-70^{\circ} \mathrm{C}$ 低温烘干. 其中唯有冷冻干燥法是保存生命物质最理想的方法 ${ }^{[6,28]}$. 其基本原理是在高真空状态下, 利用升华原理, 
使预先冻结的样品中的水份, 不经过冰的融化, 直接升华为水蒸汽以使样品干燥. 真空冷冻干燥产品确 保了生物样品中蛋白质、维生素等不变化, 易挥发热敏性成份不损失 ${ }^{[52]}$. 因而能最大限度地保持原有的 物质成份有效地防止干燥过程中的氧化, 物质成份的转化和状态变化. 冻干制品成海绵状、无干缩、含 水份极少, 因而能在常温下较长时间的穴藏保存. 但由于冷冻干燥的成本较高, 而且受到仪器设备的限 制, 所以在同位素分析中普遍采用的是 $40-70^{\circ} \mathrm{C}$ 低温烘干 ${ }^{[1,19-20]}$, 也有研究采用 $100^{\circ} \mathrm{C}$ 烘 $48 \mathrm{~h}^{[53]}$. Carabel ${ }^{[28]}$ 将食物网主要组成成分分别用冷冻干燥和 $60^{\circ} \mathrm{C}$ 低温干燥进行了比较分析, 发现两种干燥方法没有引起 $\delta^{13} \mathrm{C}$ 的显著差异，而 $\delta^{15} \mathrm{~N}$ 只在 13 个处理样品中的 2 个发现了显著差异.

\section{5 结语}

综上所述, 虽然相当的研究也进行了方法学研究, 但是, 至今还没有形成与不同生物种类相对应的 标准预处理方法. 事实上, 引起生物稳定性碳氮同位素比值精度主要来自两个方面, 即生物自身和处理 过程. 由于某些生物自身组织结构的特点, 脂类 ${ }^{[40,43]}$ 、碳酸盐含量较高 ${ }^{[6,8,28,51]}$, 微型生物个体较小, 很难 将它们同其它生物和非生命碎屑分开 ${ }^{[49]}$; 在必要的处理过程中又不可避免引起肌体其它有效组织成分的 流失 ${ }^{[6,9,28,42,50]}$, 或非目的成分的引人 ${ }^{[5,29,34-35]}$. 所以, 知道了可能引起同位素比值的原因, 在预处理过程 中既除去杂质的干扰又减少有效成分的损失是应该遵循的原则.

鉴于此，总结前人的研究结果，可以初步认为：根据研究目的的不同，采取一定的措施有效获取目 的样品, $-20^{\circ} \mathrm{C}$ 冷冻冷藏. 用福尔马林、乙醇等保存的样品, 施以校正系数, 对于长时间尺度上食物网的重 建也是可行的. 酸化是去除无机碳酸盐对有机质 $\delta^{13} \mathrm{C}$ 干扰的必要步骤. 对于碳酸盐含量较少的样品, 如 海草、鱼类肌肉组织、桡足类生物、POM滤膜等可以不用酸化或干燥后用 $12 \mathrm{~mol} / \mathrm{L}$ 浓盐酸熏蒸 $4 \mathrm{~h}$; 而对于 无机碳含量较高的样品, 如SOM、含有钙质结构的无脊椎生物、部分浮游生物等需要用 $1 \mathrm{~mol} / \mathrm{L}$ 稀盐酸滴 加的方法处理, 直至没有气泡产生, 酸化后不需要用去离子水清洗至中性. 对于生物样品 $\delta^{15} \mathrm{~N}$ 的测定则 不需要酸化处理. 最后, 样品经冷冻干燥或 $40-70^{\circ} \mathrm{C}$ 低温烘干以备稳定同位素的测定.

\section{6 参考文献}

[1] Kritzberg ES, Cole JJ, Pace ML et al. Autochthonous versus allochthonous carbon sources of bacteria: results from whole-lake ${ }^{13} \mathrm{C}$ addition experiments. Limnology and Oceanography, 2004, 49(2): 588-596.

[2] Hansson LA, Tranvik LJ. Food webs in sub-Antarctic lakes: a stable isotope approach. Polar Biology, 2003, 26: 783-788.

[3] McCallister SL, Bauer JE, Cherrier JE et al. Assessing sources and ages of organic matter supporting river and estuarine bacterial production: A multiple-isotope $\left(\delta^{14} \mathrm{C}, \delta^{13} \mathrm{C}\right.$, and $\left.\delta^{15} \mathrm{~N}\right)$ approach. Limnology and Oceanography, 2004, 49(5): 1687-1702.

[4] Peterson BJ, Fry B. Stable isotopes in ecosystem studies. Annual Review of Ecology Systematics, 1987, 18: $293-320$.

[5] Sarakinos HC, Johnson ML, Zanden MJV. A synthesis of tissue-preservation effects on carbon and nitrogen stable isotope signatures. Canadian Journal of Zoology, 2002, 80: 381-387.

[6] Bosley KL, Wainright SC. Effects of preservatives and acidification on the stable isotope ratios $\left({ }^{15} \mathrm{~N}:{ }^{14} \mathrm{~N},{ }^{13} \mathrm{C}:{ }^{12} \mathrm{C}\right)$ of two species of marine animals. Canadian Journal of Fisheries and Aquatic Sciences, 1999, 56: 2181-2185.

[7] Kaehler S and Pakhomov EA. Effects of storage and preservation of the $\delta^{13} \mathrm{C}$ and $\delta^{15} \mathrm{~N}$ signatures of selected marine organisms. Marine Ecology Progress Series, 2001, 219: 299-304.

[8] Lorrain A, Savoye N, Chauvaud L et al. Decarbonation and preservation method for the analysis of organic C and N contents and stable isotope ratios of low-carbonated suspended particulate material. Analytica Chimica Acta, 2003, 491: 125-133.

[9] Jacob U, Mintenbeck K, Brey T et al. Stable isotope food web studies: for standardized sample treatment. Marine Ecology Progress Series, 2005, 287: 251-253.

[10] 李忠义, 金显仕, 庄志猛等. 稳定同位素技术在水域生态系统研究中的应用. 生态学报, 2005, 25(11): 3052-3060.

[11] Edwards MS, Turner TF, Sharp ZD. Short- and long-term effects of fixation and preservation on stable isotope values $\left(\delta^{13} \mathrm{C}\right.$, $\left.\delta^{15} \mathrm{~N}, \delta^{34} \mathrm{~S}\right)$ of fluid-preserved museum specimens. Copeia, 2002, 4: 1106-1112.

[12] Vander Zanden MJ, Chandra S, Allen BC et al. Historical food web structure and restoration of native aquatic communities in 
the lake Tahoe (California-Nevada) basin. Ecosystems, 2003, 6: 274-288.

[13] Arrington DA, Winemiller KO. Preservation effects on stable isotope analysis of fish muscle. Transactions of the American Fisheries Society, 2002, 131: 337-342.

[14] DeNiro MJ, Epstein S. Mechanism of carbon isotope fractionation associated with lipid synthesis. Science, 1977, 197: 261-263.

[15] Mullin MM, Rau GH, Eppley RW. Stable nitrogen isotopes in zooplankton: some geographic and temporal variations in the North Pacific. Limnology and Oceanography, 1984, 29: 1267-1273.

[16] Hobson KA, Gibbs HL, Gloutney ML. Preservation of blood and tissue samples for stable-carbon and stable-nitrogen isotope analysis. Canadian Journal of Zoology, 1997, 75: 1720-1723.

[17] Rau GH, Teyssie JL, Rassoulzadegan SW et al. ${ }^{13} \mathrm{C} /{ }^{12} \mathrm{C}$ and ${ }^{15} \mathrm{~N} /{ }^{14} \mathrm{~N}$ variations among size-fractionated marine particles: implications for their origin and trophic relationships. Marine Ecology Progress Series, 1990, 59: 33-38.

[18] Lin XJ. Seasonal variation of chemical hydrography in the Southern Penghu Channel. Gao Xiong: National Sun Yat-Sen University, 2003: 21.

[19] Cole JJ, Carpenter SR, Kitchell JF et al. Pathways of organic carbon utilization in small lakes: Results from a whole-lake ${ }^{13} \mathrm{C}$ addition and coupled model. Limnology and Oceanography, 2002, 47(6): 1664-1675.

[20] Pace ML, Cole JJ, Carpenter SR et al. Whole-lake carbon-13 additions reveal terrestrial support of aquatic food webs. Nature, 2004, 427: 240-243.

[21] Atekwana EA, Krishnamurthy RV. Seasonal variation of dissolved inorganic carbon and $\delta^{13} \mathrm{C}$ of surface waters: application of a modified gas evolution technique. Journal of Hydrology, 1998, 205: 265-278.

[22] Dafner EV, Wangersky PJ. A brief overview of modern directions in marine DOC studies part II - Recent progress in marine DOC studies. Journal Environmental Monitoring, 2002, 4: 55-69.

[23] Dafner EV, Wangersky PJ. A brief overview of modern directions in marine DOC studies part I -Methodological aspects. Journal Environmental Monitoring, 2002, 4: 48-54.

[24] Raymond PA, Bauer JE. Use of ${ }^{14} \mathrm{C}$ and ${ }^{13} \mathrm{C}$ natural abundances for evaluating riverine, estuarine, and coastal DOC and POC sources and cycling: a review and synthesis. Organic Geochemistry, 2001, 32: 469-485.

[25] Wangersky PJ. Dissolved organic carbon methods : a critical review. Marine Chemistry, 1993, 41: 61-74.

[26] Sharp JH, Benner R, Bennett CA et al. Analyses of dissolved organic carbon in seawater: The JGOFS EQPAC methods comparison. Marine Chemistry, 1995, 48: 91-108.

[27] Loh AN, Bauer JE. Distribution, partitioning and fluxes of dissolved and particulate organic C, N and P in the eastern North Pacific and Southern Oceans. Deep-Sea Research I, 2000, 47: 2287-2316.

[28] Carabel S, Domínguez EG, Verísimo P et al. An assessment of sample processing methods for stable isotope analyses of marine food webs. Journal of Experimental Marine Biology and Ecology, 2006, 336: 254-261.

[29] McCallister SL, Bauer JE, Canuel EA. Bioreactivity of estuarine dissolved organic matter: A combined geochemical and microbiological approach. Limnology and Oceanography, 2006, 51(1): 94-100.

[30] Benner R, Biddanda B, Black B et al. Abundance, size distribution and stable carbon and nitrogen isotopic compositions of marine organic matter isolated by trangential-flow ultrafiltration. Marine Chemistry, 1997, 57: 243-263.

[31] Azam F, Fenchel T, Field GJ et al. The ecological role of water column microbes in the sea. Marine Ecology Progress Series, 1983, 10: 257-263.

[32] Coffin RB, Fry B, Peterson BJ et al. Carbon isotopic compositions of estuarine bacteria. Limnology and Oceanography, 1989, 34(7): 1305-1310

[33] Waichman AV. Autotrophic carbon sources for heterotrophic bacterioplankton in a floodplain lake of central Amazon. Hydrobiologia, 1996, 341: 27-37.

[34] Coffin RB, Velinsky DJ, Devereux R et al. Stable isotope analysis of nucleic acids to trace sources of dissolved substrates used by estuarine bacteria. Environmental Microbiology, 1990, 56: 2012-2020.

[35] Pelz O, Cifuentes LA, Hammer BT et al. Tracing the assimilation of organic compounds using $\delta^{13} \mathrm{C}$ analysis of unique amino 
acids in the peptidoglycan cell wall. Microbiology Ecology, 1998, 25: 229-240.

[36] Boschker HTS, Brouwer JFC, Cappenberg TE. The contribution of macrophyte-derived organic matter to microbial biomass in salt-marsh sediments: Stable carbon isotope analysis of microbial biomarkers. Limnology and Oceanography, 1999, 44: 309-319.

[37] 杨宇峰, 王 庆, 陈菊芳等. 河口浮游动物生态学研究进展. 生态学报, 2006, 26(2): 576-585.

[38] Schneider JC ed. Manual of fisheries survey methods II : with periodic updates. Lansing: Michigan Department of Natural Resources, 2000

[39] 黄祥飞，陈伟民，蔡启铭等. 湖泊生态调查观测与分析. 北京：中国标准出版社, 2000: 86.

[40] Hobson KA, Fisk Aaron, Karnovsky $\mathrm{N}$ et al. A stable isotope $\left(\delta^{13} \mathrm{C}, \delta^{15} \mathrm{~N}\right)$ model for the North Water food web: implications for evaluating trophodynamics and the flow of energy and contaminants. Deep-Sea Research II, 2002, 49: 5131-5150.

[41] Malseed T. Stable isotope analysis of the food web supporting Sardinops sagax in the waters off Esperance, Western Austualia. Perth: The University of Western Australia, 2004: 35-36.

[42] Bunn SE, Loneragan NR, Kempster MA. Effects of acid washing on stable isotope ratios of C and N in penaeid shrimp and seagrass: implications for food-web studies using multiple stable isotopes. Limnology and Oceanography, 1995, 40: 622-625.

[43] Schlechtriem Ch, Focken U, Becker K. Effect of different lipid extraction methods on the delta13C of lipid-free fractions of fish and different fish feeds. Isotopes in Environmental and Health Studies, 2003, 39(2): 135-140

[44] Murry BA, Farrell JM, Teece MA et al. Effect of lipid extraction on the interpretation of fish community trophic relationships determined by stable carbon and nitrogen isotopes. Canadian Journal of Fisheries and Aquatic Sciences, 2006, 63(10): 2167-2172.

[45] Currin CA, Newell SY, Paerl HW. The role of benthic microalgae and standing dead Spartina alterniflora in salt marsh food webs: implications based on multiple stable isotope analysis. Marine Ecology Progress Series, 1995, 121: 99-116.

[46] Pel R, Hoogveld H, Floris V. Using the hidden isotopic heterogeneity in phyto- and zooplankton to unmask disparity in trophic carbon transfer. Limnology and Oceanography, 2003, 48: 2200-2207.

[47] Bidigare RR, Kennicutt MCI, Macko SA. Isolation and purification of chlorophylls a and b for the determination of stable carbon and nitrogen isotope compositions. Analytical Chemistry, 1991, 63: 130-133.

[48] Rolff C. Seasonal variation in $\delta^{13} \mathrm{C}$ and $\delta^{15} \mathrm{~N}$ of size-fractionated plankton at a coastal station in the northern Baltic proper. Marine Ecology Progress Series, 2000, 203: 47-65.

[49] Hamilton SK, Sippel SJ, Bunn SE. Separation of algae from detritus for stable isotope or ecological stoichiometry studies using density fractionation in colloidal silica. Limnology and Oceanography: Methods 3, 2005: 149-157.

[50] Goering J, Alexander V, Haubenstock N. Seasonal variability of stable carbon and nitrogen isotope ratios of organism in a North Pacific Bay. Estuarine, Coastal and Shelf Science, 1990, 30: 239-260.

[51] Bunn SE, Loneragan NR, Kempster MA. Effects of acid washing on stable isotope ratios of C and N in penaeid shrimp and seagrass: implications for food-web studies using multiple stable isotopes. Limnology and Oceanography, 1995, 40: 622-625.

[52] Abdelwahed W, Degobert G, Stainmesse S et al. Freeze-drying of nanoparticles: formulation, process and storage considerations. Advanced Drug Delivery Reviews, 2006, 58: 1688-1713.

[53] Pruell RJ, Taplin BK, Lake JL et al. Nitrogen isotope ratios in estuarine biota collected along a nutrient gradient in Narragansett Bay, Rhode Island, USD. Marine Pollution Bulletin, 2006, 52: 612-620. 